\title{
Interleukin-4 protects from chemotherapy-induced peripheral neuropathy in mice modal via the stimulation of IL-4/STAT6 signaling ${ }^{1}$
}

\author{
Qingbin Shi', Xiuying Cai", Guixiang Shi"l', Xingle Lv", Jinping Yuv, Feng Wang ${ }^{\mathrm{VI}}$
}

'MD, Doctor-in-charge, Department of Encephalopathy, Zhangqiu District, Traditional Chinese Medicine Hospital, China. Acquisition, analysis and interpretation of data; manuscript preparation.

"MD, Doctor-in-charge, Department of Encephalopathy, Zhangqiu District, Traditional Chinese Medicine Hospital, China. Technical procedures, acquisition of data.

'"MD, Nurse-in-charge, Department of Encephalopathy, Zhangqiu District, Traditional Chinese Medicine Hospital, China. Technical procedures.

IvMD, Nurse practitioner, Department of Encephalopathy, Zhangqiu District, Traditional Chinese Medicine Hospital, China. Technical procedures.

${ }^{\vee} M D$, Physician, Department of Encephalopathy, Zhangqiu District, Traditional Chinese Medicine Hospital, China. Technical procedures.

V'MD, Chief Physician, Department of Encephalopathy, Zhangqiu District, Traditional Chinese Medicine Hospital, China. Conception and design of the study, manuscript preparation, final approval.

\begin{abstract}
Purpose: To investigate the possible role of IL-4 signaling pathway in vincristine-induced peripheral neuropathy.

Methods: The mouse model of vincristine-induced peripheral neuropathy and interleukin (IL)-4 knockout mice were utilized to investigate the possible role of IL-4 signaling pathway in vincristine-induced peripheral neuropathy. Vincristine induced increased sensitivity to mechanical stimulation was measured by von Frey hair test 7 and 14 days after intraperitoneal administration of $0.1 \mathrm{mg} / \mathrm{kg}$ vincristine in mice. Relative expression levels of cytokines were detected by quantitative real-time PCR. STAT6 expression following vincristine treatment was assessed with western blotting.

Results: We discovered that IL-4/STAT6 signaling was down-regulated in vincristine-treated mice. Deletion of IL-4 in mice increased the sensitivity to mechanical allodynia. IL-4 knockout mice also produced more pro-inflammatory cytokines, including IL-1 $\beta$ and TNF- $\alpha$. Notably, coadministration of exogenous recombination IL-4 significantly prevented vincristine-induced mechanical allodynia.

Conclusion: Anti-inflammatory cytokine IL-4 protects rodent model from vincristine-induced peripheral neuropathy via the stimulation of IL-4/STAT6 signaling and inhibition of the proinflammatory cytokines.
\end{abstract}

Key words: Interleukin-4. Drug Therapy. Peripheral Nervous System Diseases. Mice. 


\section{Introduction}

Chemotherapeutic drugs such as vincristine, paclitaxel, andoxaliplatin, are widely used to treat several types of malignant tumors. Nevertheless, their clinical use are also accompanied by severe side effects, including peripheral neuropathy and chronic neuropathic pain ${ }^{1}$. The vinca alkaloid compound vincristine (VCR), which was originally derived from the madagascar periwinkle plant, is a common chemotherapeutic agent for a variety of malignancies including acute lymphoblastic leukemia, lymphomas, sarcomas, neuroblastoma, and kidney, liver, lung, brain and breast tumors amongst others ${ }^{2,3}$. However, vincristine treatment is limited by a progressive peripheral neuropathy, such as paresthesia and dysesthesia. Vincristine-induced peripheral neuropathy (VIPN), which affects sensory, motor, and autonomic nerves, is often resistant to standard analgesics ${ }^{4-6}$. To date, rodent and cell models of VIPN have been developed to elucidate the underlying mechanisms ${ }^{1,7}$, but the exact mechanism is still not completely understood.

It is now known that chemotherapeutic exposure could enhance the release of different cytokines. There is evidence that administration of chemotherapeutic drugs such as vincristine, paclitaxel and cisplatin could lead to the increased pro-inflammatory cytokines and chemokines such as TNF- $\alpha$, IL-1 $\beta$ and $\mathrm{MCP}-1^{7}$.These pro-inflammatory cytokines can result in inflammation-related responses, for instance, the release of histamine ${ }^{8}$, and can bind to their receptors to enhance activity in neuropathy pain pathways ${ }^{9}$. On the other hand, anti-inflammatory cytokines also participate in peripheral neuropathy. Previous studies have shown that $\mathrm{CD} 4^{+}$regulatory Tcells (Tregs), which can produce anti-inflammatory cytokines including interleukin (IL)-4, IL10 , and transforming growth factor TGF- $\beta$, plays important role in endogenous recovery from neuropathy-induced pain ${ }^{10}$. Peripheral depletion of Tregs in mice resulted in prolonged mechanical pain hypersensitivity. Furthermore, there is evidence that endogenous antiinflammatory cytokinelL-10 are required for resolution of chemotherapy-induced neuropathic pain ${ }^{11}$. Exogenous administration of IL-10 and IL-4 could suppress allodynia in neuropathic animal models, reducing the production of pro-inflammatory cytokines, microglia responses and pain behavior ${ }^{9,12-14}$. When rats were pre-treated withIL-4, the pain responses were attenuated, and the onset of pain hypersensitivity was delayed ${ }^{15-17}$. All of these indicating that anti-inflammatory cytokine IL-4 have potential links with neuropathy pain.

Although experimental evidences have suggested that IL-4 may play a protective role in peripheral neuropathy, little is known about the exact underlying mechanism. Whether IL-4 is participate in chemotherapy-induced peripheral neuropathy is still unknown. Thus, in the present study, we utilize a mouse model of vincristine-induced peripheral neuropathy and IL-4 knockout mice to investigate the possible role of IL-4 signaling pathway in vincristineinduced peripheral neuropathy.

\section{- Methods}

The experiments with mice were in full compliance with the European Communities Council Directive of 24 November 1986 (86/609/EEC) or with the Guidelines laid down by the NIH in the US.

Male wild-type (WT) C57BL/6Jand interleukin (IL)-4 knockout (KO) mice (with C57BL background) weighing30-35 g were used throughout this study. Mice were obtained from the Jackson Laboratory (Bar Harbor, ME). Rodents were housed under conditions of optimum light, temperature and humidity (12 h light-dark cycle, $22 \pm 3^{\circ} \mathrm{C}, 60-80 \%$ humidity), and had ad libitum access to food and water. 
All experimental procedures were performed according to the Guidelines for Animal Care and Use of Zhangqiu District of Traditional Chinese Medicine Hospital (NO.20160163).

\section{Vincristine treatment}

The chemotherapeutic drug vincristine was purchased from Novopharm (Nippon Kayaku, Tokyo, Japan). Vincristine was diluted in water at the concentration of $1.0 \mathrm{mg} /$ $\mathrm{mL}$, and then diluted in sterile saline before intraperitoneally injections. One group of mice received saline served as the normal control. The other group received vincristine at a dosage of $0.1 \mathrm{mg} / \mathrm{kg}$. The injections were performed from day 0 to day 4 , followed by 2 days of rest, and a second round of injection from day7 to day 11 .

von Frey hair test

Mechanical threshold testing was performed on day0 before vincristine administration and on day14 after vincristine treatment. The mice were placed in a clear plastic box $(23 \times 23 \times 12 \mathrm{~cm})$ with a mesh floor. The animals were acclimatized for 30 min before behavioral testing. Subsequently, a series of von Frey hairs $(2,4,8 \mathrm{~g})$ were applied perpendicular to the midplantar surface of hindpaw. Each mouse received five-second stimulations for 5 times. A sharp withdrawal of the stimulated region was regarded as a positive response. The number of withdrawal responses were examined, and the overall with drawal frequency was calculated for each group.

\section{Western blotting}

Samples were lysed in RadiolmmunoPrecipitation Assay (RIPA) buffer (50 mmol/L Tris $\mathrm{HCl}$ (pH 8.0), $150 \mathrm{mmol} / \mathrm{L} \mathrm{NaCl}$, $1 \%$ NP-40, $0.5 \%$ sodium deoxycholate, and $0.1 \%$ sodium dodecyl sulfate [SDS]) with protease inhibitor cocktail (Roche) and 1 mMPMSF for $30 \mathrm{~min}$ on ice. After centrifugation at 14,000 $\mathrm{g}$ for $15 \mathrm{~min}$ at $4 \mathrm{C}$, the lysates were boiled in $4 \times$ SDS loading buffer. Equal amounts of protein (20 mg/lane) were separated by SDS-PAGE, transferred to a PVDF membrane, and detected by immunoblotting analysis with antibodies using Immobilon Western Chemiluminescent HRP Substrate (Millipore).

\section{Quantitative Real-Time PCR}

Total RNA was extracted from samples with TRIzolreagent (Invitrogen) and reversetranscribed using the SuperScript III reverse transcriptase protocol (Invitrogen). Quantitative real-time PCR was performed using $A B I Q 7$ Real-Time PCR system (Applied Biosystems). Relative expressions of mRNAs were calculated using the comparative $\mathrm{Ct}$ method $^{18}$, and were normalized to housekeeping gene GAPDH.

\section{Statistical analysis}

Data are expressed as mean \pm SEM. Statistical analysis was performed using SPSS22.0and GraphPad Prism 5.0 softwares. One-way ANOVA or repeated-measures t test followed by Bonferroni post hoc analysis were applied. Differences with $p<0.05$ were defined as the threshold for significance.

\section{- Results}

\section{Vincristine-induced peripheral neuropathy in mice}

Wild-type (WT) C57BL/6J mice were intraperitoneally (i.p.) treated with two rounds of vincristine daily at a dosage of $0.1 \mathrm{mg} / \mathrm{kg}$ for 5 days, with 2 days rest in between (Figure $1 \mathrm{~A}$ ). Mechanical threshold testing was performed on day0 (before vincristine administration), day7 (after a round of vincristine treatment) and day14 (after two rounds of vincristine 
treatment). The results showed that withdrawal frequency of Von Frey hair test for mechanical allodynia was almost the same between two groups on day0. However, on day7 and day14, vincristine-treated mice expressed higher withdrawal frequency as compared to salinetreated animals (Figure 1B). That means chemotherapeutic drug vincristine could lead to peripheral neuropathy in animal models.

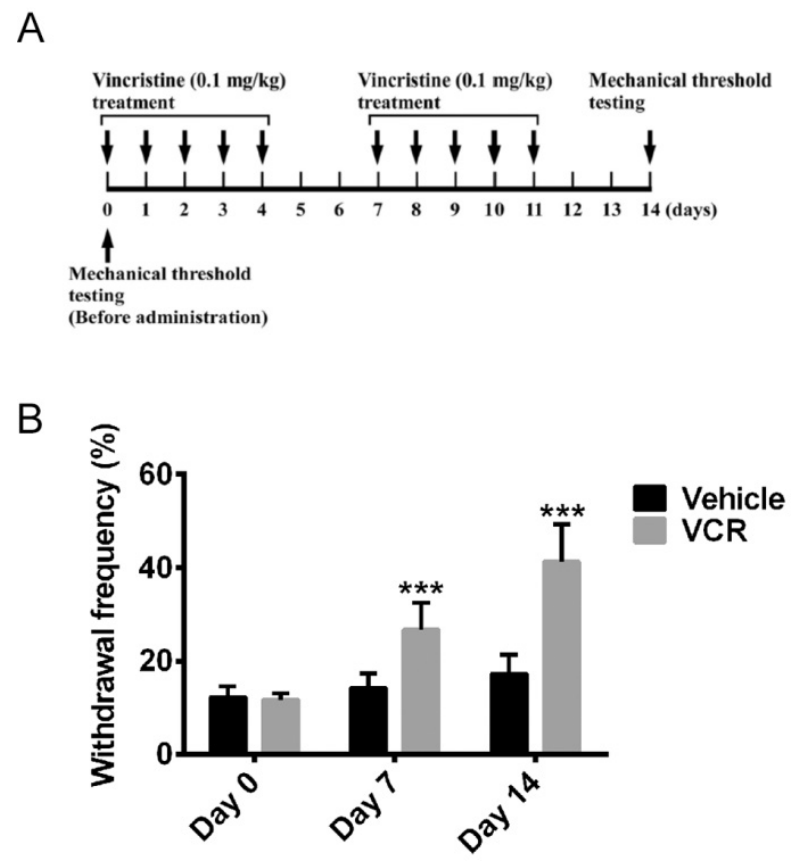

Figure 1 - Effect of vincristine-induced peripheral neuropathy in Mice. (A) Vincristine treatment schedule; arrows: vincristine injection. (B) Hind paw withdrawal responses to von Frey hair test were measured after administration of vincristine or saline. Graphs are expressed as mean \pm SEM (one-way ANOVA followed by Bonferroni's post hoc test; $\left.{ }^{* * *} p<0.001\right)$.

\section{IL-4/STAT6 signaling was down-regulated in vincristine-treated mice}

In order to investigate the role of IL-4/STAT6 signaling in vincristine-treated animals, IL-4 mRNA relative expression level was measured in sciatic nerves. Samples were collected on day14, when behavioral test expressed the most significant difference between vehicle and vincristine-treated groups. The results showed that endogenous IL-4 mRNA relative expression level was downregulated in vincristine-treated mice compared to vehicle group (Figure 2A). Furthermore, western blotting result showed that p-STAT6, the downstream effector of IL-4, was also downregulated in vincristine-treated mice sciatic nerves (Figure 2B). These results indicating that chemotherapeutic drug vincristine may contribute to peripheral neuropathy by downregulating anti-inflammatorylL-4 pathway.
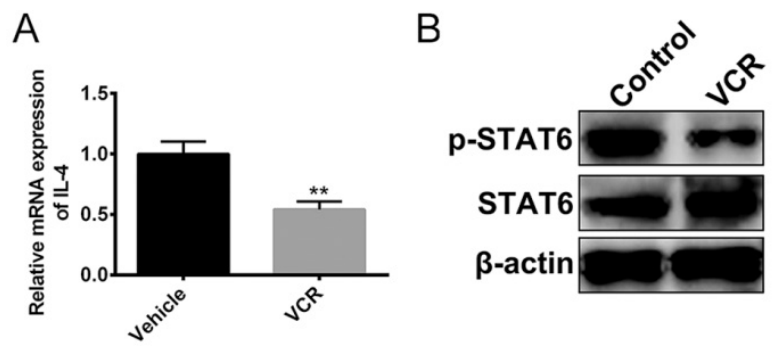

Figure 2 - IL-4/STAT6 signaling was down-regulated in vincristine-treated mice. (A) Quantitative realtime PCR showed that endogenous IL-4 mRNA relative expression level was down-regulated in vincristine-treated mice compared to vehicle group. Relative expressions of mRNAs were normalized to housekeeping gene GAPDH. (B) p-STAT6 protein level was down-regulated in vincristine-treated mice sciatic nerves. Graphs are expressed as mean \pm SEM, ${ }^{* *} p<0.01$.

\section{Deletion of IL-4 acceleratedmechanical allodynia and pro-inflammatory cytokines production}

To further discover the necessity of IL-4 signaling pathway in mechanical allodynia, IL-4 knockout (KO) mice were used for von Frey hair test. As a result, IL-4 KO mice expressed higher withdrawal frequency as compared to WT group (Figure 3A). Next, IL-4 was re-introduced into IL-4 KO mice by injection of recombination IL-4 protein. On day7 and day14 after IL-4 protein injection, the withdrawal frequency were significantly decreased compared to 
IL-4 KO group (Figure 3A). It is reported that the protective function of IL-4 was often associated with decreased production of proinflammatory cytokines, such as IL-1ßand TNF- $\alpha^{19-21}$. Thus, we measured the relative expression level of IL-1ßand TNF- $\alpha$ in WT, IL-4 $\mathrm{KO}$ and IL-4 KO + IL-4 mice. It showed that these two pro-inflammatory cytokines were over expressed in IL-4 KO mice compared to WT mice (Figure $3 \mathrm{~B}-\mathrm{C}$ ). Notably, reduced expression of IL-1ßand TNF- $\alpha$ were discovered when IL-4 was re-introduced into IL-4 KO mice (Figure $3 \mathrm{~B}-\mathrm{C}$ ). In addition, re-introduction of recombination IL-4 protein attenuated IL-4 KOinduced p-STAT6 down-regulation (Figure 3D).
A
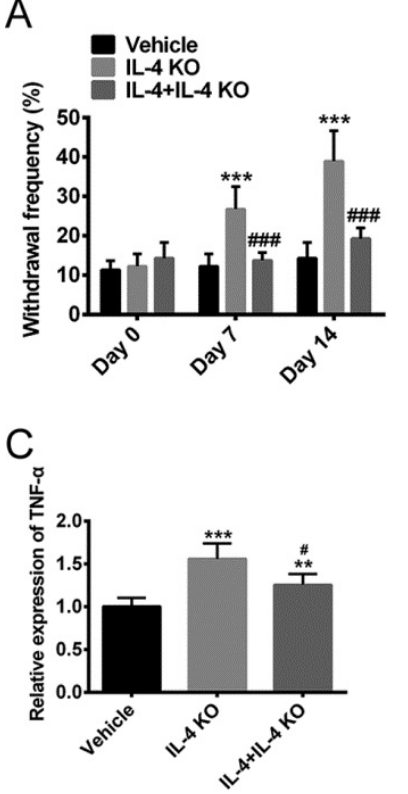

B
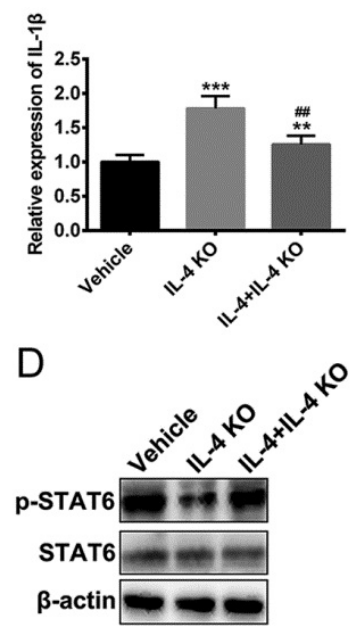

Figure 3 - Deletion of IL-4 accelerated mechanical allodynia and pro-inflammatory cytokines production in mice. (A) Hind paw withdrawal responses to von Frey hair test were measured. Vehicle: WT mice; IL-4 KO: IL-4 knockout mice; L-4 $\mathrm{KO}+\mathrm{IL}-4$ : injection of recombination IL-4 protein into IL-4 knockout mice. (B) and (C) Quantitative real-time PCR of IL-1ßand TNF- $\alpha$. (D) p-STAT6 protein level was measured by western blotting. Graphs are expressed as mean \pm SEM (one-way ANOVA followed by Bonferroni's post hoc test). ** $p<0.01, * * * p<0.001$ vs. vehicle; \# $p<0.05$, \#\# $\mathrm{p}<0.01$, \#\#\# $\mathrm{p}<0.001$ vs. IL-4 KO.

\section{IL-4 attenuated vincristine-induced peripheral neuropathy}

Knowing that IL-4 is necessary for reducing mechanical allodynia, we next investigated whether administration of exogenous IL-4could suppress vincristineinduced peripheral neuropathy in mice. We found out that there were significant reductions of withdrawal frequency in mice with exogenous IL-4treatment when exposed to vincristine on day7 and day14 (Figure 4A).

p-STAT6 protein level was significantly down-regulated in vincristine-treated mice compared to vehicle group as shown previously in Figure 2B. Accordingly, p-STAT6 protein level was restored in exogenous IL-4-treated mice (Figure $4 \mathrm{~B})$. It indicating that activation of IL-4/STAT6 signaling pathway could attenuated vincristineinduced peripheral neuropathy in mice.

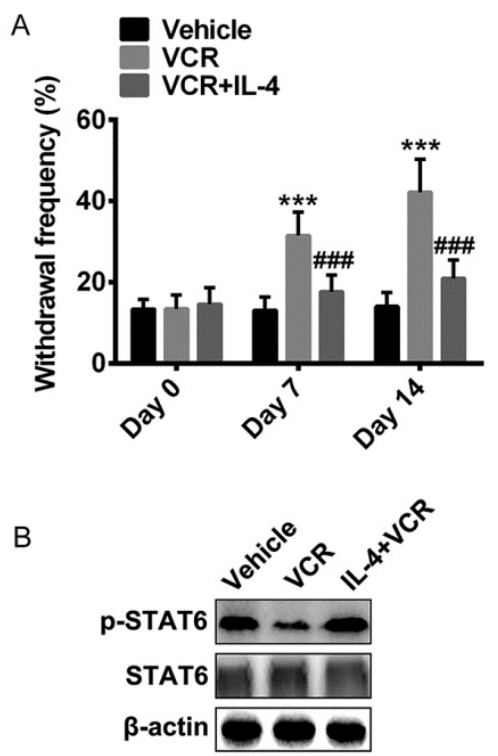

Figure 4 - IL-4 attenuated vincristine-induced peripheral neuropathy in mouse models. (A) Hind paw withdrawal responses to von Frey hair test were measured. Vehicle: WT mice; VCR: WT mice treated with vincristine; VCR+IL-4: injection of recombination IL-4 protein into vincristine-treated mice. (B) STAT6 protein level was measured by western blotting. Graphs are expressed as mean \pm SEM (one-way ANOVA followed by Bonferroni's post hoc test). ${ }^{* * *} \mathrm{p}<0.001$ vs. vehicle; \#\#\# $\mathrm{p}<$ 0.001 vs. VCR. 


\section{- Discussion}

The vinca alkaloid compound vincristine has successfully become a chemotherapeutic agent for a variety of malignancies since 1950 's ${ }^{3}$. It binds to the $\beta$-subunit of tubulin heterodimers to prevent the polymerization and incorporation of microtubules. Thus, dividing cells were arrested in metaphase ${ }^{22}$. Due to the fact that $\beta$-subunit of microtubules are also critical components of nerve fiber axons, vincristine treatment can cause peripheral neuropathy as well ${ }^{23,24}$. The peripheral nervous system is frequently affected by vincristine treatment, leading to severe peripheral neurotoxicity that includes neuropathic pain and autonomic impairment ${ }^{25}$. Moreover, vincristine-induced peripheral neuropathy may not resolve over time, which not only affects quality of life for years, but also contributes to drug dose reductions ${ }^{26}$. At present, knowledge about the mechanisms underlying vincristine-induced peripheral neuropathy remains obscure. Therefore, it is highly imperative to explore the related molecular and signaling pathways.

In this study, we found that antiinflammatory cytokine IL-4 protects from vincristine-induced peripheral neuropathy via the stimulation of IL-4/STAT6 signaling. IL-4/STAT6 signaling was proved to be downregulated in vincristine-treated mice. By performing von Frey hair test, we discovered that deletion of IL-4 accelerated mechanical allodynia in animal models. IL-4 knockout mice also produced more pro-inflammatory cytokines, including IL-1ßand TNF- $\alpha$. Furthermore, peripheral neuropathy was attenuated when exogenous recombination IL-4 was re-introduced into vincristine-treated mice. These findings demonstrated thatIL-4/ STAT6 signaling plays a protective role against vincristine-induced peripheral neuropathy.

Several previous studies have demonstrated the role of neuro-immune balance in neuropathic pain ${ }^{27}$. However, they mainly focused on the pro-inflammatory cytokines such as TNF, IL-1 $\beta$ and IL-6 $6^{28}$. To our knowledge, this is the first study to discover the potential protective role of IL-4 in vincristineinduced peripheral neuropathy. In our study, anti-inflammatory cytokine IL-4 was found to be down-regulated in vincristine-treated mice, while pro-inflammatory cytokinesIL-1ßand TNF- $\alpha$ were up-regulated. It is in consistent with the previous report that patients with complex regional pain syndrome and painful neuropathy have increased levels of pro-inflammatory cytokines TNF, IL-2 and IL-6 and decreased levels of anti-inflammatory cytokines, IL-10 and IL- $4^{29,30}$. Others have shown that IL-4, often released by activated $T$ cells, mast cells and granulocytes, could inhibit the production of TNF, IL-1 $\beta$ and IL- $6^{15}$. Collectively, our findings indicating that IL-4 protects against vincristineinduced peripheral neuropathy by reducing the release of pro-inflammatory cytokines.

This study also had some limitations. First, neurotoxicity often depends on the type of drug used and the total cumulative dose ${ }^{31}$. It is possible that different conditions may share common pathophysiology and may due to the imbalance of cytokine network. In this study, vincristine treatment only had one dosage of $0.1 \mathrm{mg} / \mathrm{kg}$. The downstream effect and animal behavior of different cumulative dose has not been examined. Whether the protected role of IL-4 signaling is a common event in chemotherapy-induced peripheral neuropathy remains unknown. In addition, the reason of IL-4 down-regulation upon vincristine treatment is still unclear because only mRNA level of IL-4 was measured. It is supposed to have reduced transcriptional activity, and further study is still needed.

As a protective molecular in vincristineinduced peripheral neuropathy, IL-4 may become a potential therapeutic target. Modulation of cytokine signaling by promoting anti-inflammatory cytokines and blocking pro-inflammatory cytokines may become treatment strategies for chemotherapyinduced peripheral neuropathy in the future. 


\section{Conclusion}

The anti-inflammatory cytokine IL-4 protects rodent model from vincristine-induced peripheral neuropathy via the stimulation of IL-4/STAT6 signaling and inhibition of the proinflammatory cytokines.

\section{References}

1. Carozzi VA, Canta A, Chiorazzi A. Chemotherapy-induced peripheral neuropathy: what do we know about mechanisms? Neurosci Lett. 2015;596:90107. doi: 10.1016/j.neulet.2014.10.014.

2. Bradley WG, Lassman LP, Pearce GW, Walton $J N$. The neuromyopathy of vincristine in man. Clinical, electrophysiological and pathological studies. J Neurol Sci. 1970;10(2):107-31. PMID: 4314181.

3. Johnson IS, Armstrong JG, Gorman M, Burnett JP Jr. The vinca alkaloids: a new class of oncolytic agents. Cancer Res. 1963;23:1390-427. PMID: 14070392.

4. Gomber S, Dewan P, Chhonker D. Vincristine induced neurotoxicity in cancer patients. Indian J Pediatr. 2010;77(1):97-100. doi: 10.1007/s12098-009-0254-3.

5. Anghelescu DL, Faughnan LG, Jeha S, Relling MV, Hinds PS, Sandlund JT, Cheng C, Pei D, Hankins G, Pauley JL, Pui CH. Neuropathic pain during treatment for childhood acute lymphoblastic leukemia. Pediatr Blood Cancer. 2011;57(7):1147-53. PMID: 3136575.

6. Argyriou AA, Bruna J, Marmiroli P, Cavaletti G. Chemotherapy-induced peripheral neurotoxicity (CIPN): an update. Crit Rev Oncol Hematol. 2012;82(1):51-77.doi: 10.1016/j.critrevonc.2011.04.012.

7. Boyette-Davis JA, Walters ET, Dougherty P.M. Mechanisms involved in the development of chemotherapy-induced neuropathy. Pain Manag. 2015;5(4):285-96. PMID: 4504016.

8. McMahon SB, Cafferty WB, Marchand F. Immune and glial cell factors as pain mediators and modulators. Exp Neurol. 2005;192(2):444-62. doi: 10.1016/j. expneurol.2004.11.001.

9. Ledeboer A, Jekich BM, Sloane EM, Mahoney $\mathrm{JH}$, Langer SJ, Milligan ED, Martin D, Maier SF, Johnson KW, Leinwand LA, Chavez RA,
Watkins LR. Intrathecal interleukin-10 gene therapy attenuates paclitaxel-induced mechanical allodynia and proinflammatory cytokine expression in dorsal root ganglia in rats. Brain Behav Immun. 2007;21(5):68698. doi: 10.1016/j.bbi.2006.10.012.

10.Austin PJ, Kim CF, Perera CJ, Moalem-Taylor G. Regulatory T cells attenuate neuropathic pain following peripheral nerve injury and experimental autoimmune neuritis. Pain. 2012;153(9):1916-31. doi: 10.1016/j. pain.2012.06.005.

11.Krukowski K, Eijkelkamp N, Laumet G, Hack CE, Li Y, Dougherty PM, Heijnen CJ, Kavelaars A. CD8+ T cells and endogenous IL-10 are required for resolution of chemotherapyinduced neuropathic pain. J Neurosci. 2016;36(43):11074-83. doi: 10.1523/ JNEUROSCI.3708-15.2016.

12.McKelvey R, Berta T, Old E, Ji RR, Fitzgerald $M$. Neuropathic pain is constitutively suppressed in early life by antiinflammatory neuroimmune regulation. J Neurosci. 2015;35(2):457-66. doi: 10.1523/ JNEUROSCI.2315-14.2015.

13. Milligan ED, Sloane EM, Langer SJ, Cruz $P E$, Chacur M, Spataro L, Wieseler-Frank J, Hammack SE, Maier SF, Flotte TR, Forsayeth JR, Leinwand LA, Chavez R, Watkins LR. Controlling neuropathic pain by adenoassociated virus driven production of the anti-inflammatory cytokine, interleukin-10. Mol Pain. 2005;1:9. doi: 10.1186/17448069-1-9.

14. Milligan ED, Watkins LR. Pathological and protective roles of glia in chronic pain. Nat Rev Neurosci. 2009;10(1):23-36. doi: 10.1038/nrn2533.

15.Cunha FQ, Poole S, Lorenzetti BB, Veiga FH, FerreiraSH.Cytokine-mediatedinflammatory hyperalgesia limited by interleukin-4. $\mathrm{Br} J$ Pharmacol. 1999;126(1):45-50. doi: 10.1038/sj.bjp.0702266.

16.Vale ML, Marques JB, Moreira CA, Rocha FA, Ferreira SH, Poole S, Cunha FQ, Ribeiro RA. Antinociceptive effects of interleukin-4, -10 , and -13 on the writhing response in mice and zymosan-induced knee joint incapacitation in rats. J Pharmacol Exp Ther. 2003;304(1):102-8. doi: 10.1124/ jpet.102.038703.

17. Hao S, Mata M, Glorioso JC, Fink DJ. HSVmediated expression of interleukin-4 in 
dorsal root ganglion neurons reduces neuropathic pain. Mol Pain. 2006;2:6. doi: 10.1186/1744-8069-2-6.

18.Schmittgen TD, Livak KJ. Analyzing real-time PCR data by the comparative $C(T)$ method. Nat Protoc. 2008;3(6):1101-8. PMID: 18546601.

19.Czimmerer Z, Daniel B, Horvath A, Ruckerl D, Nagy G, Kiss $M$, Peloquin M, Budai MM, Cuaranta-Monroy I, Simandi Z, Steiner L, Nagy B Jr, Poliska S, Banko C, Bacso Z, Schulman IG, Sauer S, Deleuze JF, Allen JE, Benko S, Nagy L. The transcription factor STAT6 mediates direct repression of inflammatory enhancers and limits activation of alternatively polarized macrophages. Immunity. 2018;48(1):75-90. e76. doi: 10.1016/j.immuni.2017.12.010.

20.Suzuki K, Nakajima H, Ikeda K, Maezawa Y, Suto A, Takatori $H$, Saito $Y$, Iwamoto I. IL-4-Stat6 signaling induces tristetraprolin expression andinhibits TNF-alpha production in mast cells. J Exp Med. 2003;198(11):171727. doi: $10.1084 /$ jem.20031701.

21. Levings MK, Schrader JW. IL-4 inhibits the production of TNF-alpha and IL-12 by STAT6dependent and -independent mechanisms. J Immunol. 1999;162(9):5224-9. PMID: 21211639.

22.Dumontet C, Jordan MA. Microtubulebinding agents: a dynamic field of cancer therapeutics. Nat Rev Drug Discov. 2010;9(10):790-803. doi: 10.1038/nrd3253.

23.Lobert S, Vulevic B, Correia JJ. Interaction of vinca alkaloids with tubulin: a comparison of vinblastine, vincristine, and vinorelbine. Biochemistry. 1996;35(21):6806-14. doi: 10.1021/bi953037i.

24.Legha SS. Vincristine neurotoxicity.
Pathophysiology and management. Med Toxicol. 1986;1(6):421-7. PMID: 3540519.

25.Marmiroli P, Nicolini G, Miloso M, Scuteri A, Cavaletti G. The fundamental role of morphology in experimental neurotoxicology: the example of chemotherapy-induced peripheral neurotoxicity. Ital J Anat Embryol. 2012;117(2):75-97. doi: 10.13128/IJAE11756.

26. Mora E, Smith EM, Donohoe C, Hertz DL. Vincristine-induced peripheral neuropathy in pediatric cancer patients. Am J Cancer Res. 2016;6(11):2416-30. PMID: 5126263.

27.Austin PJ, Moalem-Taylor G. The neuroimmune balance in neuropathic pain: involvement of inflammatory immune cells, immune-like glial cells and cytokines. J Neuroimmunol. 2010;229(1-2): 26-50. doi: 10.1016/j.jneuroim.2010.08.013.

28.Park HJ. Chemotherapy induced peripheral neuropathic pain. Korean J Anesthesiol. 2014;67(1):4-7. doi: 10.4097/ kjae.2014.67.1.4.

29.Davies AL, Hayes KC, Dekaban GA. Clinical correlates of elevated serum concentrations of cytokines and autoantibodies in patients with spinal cord injury. Arch Phys Med Rehabil. 2007;88(11):1384-93. doi: 10.1016/j.apmr.2007.08.004.

30.Uceyler N, Eberle T, Rolke R, Birklein F, Sommer C. Differential expression patterns of cytokines in complex regional pain syndrome. Pain. 2007;132(1-2):195-205. doi: 10.1016/j.pain.2007.07.031.

31.Quasthoff S, Hartung HP. Chemotherapyinduced peripheral neuropathy. J Neurol. 2002;249(1):9-17. PMID: 10975744.

\section{Correspondence:}

Feng Wang

No.1463, Xiushui Street, Zhangqiu District

Ji'nan, Shandong, P.R. China, 250200

wangfengwydem@163.com

Received: Feb 18, 2018

Review: Apr 15, 2018

Accepted: May 14, 2018
Conflict of interest: none

Financial source: none
${ }^{1}$ Research performed at Department of Encephalopathy, Zhangqiu District of Traditional Chinese Medicine Hospital, China. 